en, die mit der medizinischen Behandlungspflege verbundenen Kosten eines ambulanten Pflegedienstes zu übernehmen. Insoweit handelt es sich aber wiederum - anders als hier - um medizinische Behandlungspflege, und im Übrigen ging das BSG davon aus, dass kein geeignetes Pflegepersonal zur Verfügung stehe. Hier hat die Einrichtung aber Pflegefachkräfte vorzuhalten, wie oben ausgeführt ist.

Die Einrichtung wird damit, ihre Aufgaben aus der LV zu erfüllen, auch nicht übermäßig belastet, denn gemäß \55 S. 2 SGB XII ist sie berechtigt, gegenüber dem Antragsgegner anzuzeigen, wenn sie feststellt, dass ein behinderter Mensch so pflegebedürftig ist, dass die Pflege in der Einrichtung nicht mehr sichergestellt werden kann. Dann müssen der Sozialhilfeträger, die zuständige Pflegekasse und der Einrichtungsträger vereinbaren, dass die Leistung in einer anderen Einrichtung erbracht wird. Eine derartige Anzeige ist aber noch nicht erfolgt. Solange das nicht der Fall ist, hat die Einrichtung für die umfassende Betreuung und Pflege des Antragstellers zu sorgen.

DOI: 10.1007/s00350-013-3497-y

\section{Anmerkung zu LSG Schlesw.-Holst., Beschl. v. 21.12.2012 - L 9 SO 151/12 B ER (SG Schleswig) Kyrill Makoski}

Erklärtes Ziel der Sozialpolitik ist die Verbesserung der Position von behinderten Menschen. Die Bundesrepublik Deutschland hat sich mit der Ratifikation des Übereinkommens über die Rechte von Menschen mit Behinderungen der Vereinten Nationen (BGBl. 2008 II, S. 1419) verpflichtet, behinderten Menschen einen Zugang zu angemessener schulischer und beruflicher Bildung sowie beruflicher Tätigkeit zu gewähren. Hierzu wurden die Sozialhilfeträger zu Leistungen der Eingliederungshilfe nach $\$ 53$ SGB XII verpflichtet. Die Eingliederungshilfe wird häufig in Einrichtungen nach $\$ 43$ a SGB XII erbracht; dies sind vor allem Schulen und Werkstätten für Behinderte. Bei der Unterbringung in einer entsprechenden Einrichtung ist grundsätzlich der vollständige Pflegebedarf des Betroffenen abgedeckt; dafür beteiligt sich ggf. die Pflegeversicherung an den Kosten.

Bisher nicht abschließend geklärt ist die Frage, ob der Sozialhilfeträger neben den Leistungen der Einrichtung zusätzlich einen ambulanten Pflegedienst für bestimmte Leistungen bezahlen muss. Diese Frage hat das LSG Schleswig-Holstein verneint. Dabei hat es darauf abgestellt, dass eine Einrichtung ausgewählt werden muss, in der der gesamte Pflegebedarf des Betroffenen gedeckt werden kann ( 555 S. 1 SGB XII). Bei geändertem Pflegebedarf ist der Betroffene in eine andere Einrichtung zu verlegen ( $\$ 55$ S. 2 SGB XII). Dabei sind die Wünsche des Betroffenen besonders zu beachten ${ }^{1}$. Sofern die Kranken- oder Pflegekasse die vom Betreiber der Einrichtung für erforderlich erachtete Verlegung nicht mittragen will, muss der Sozialhilfeträger in Vorleistung treten und die Leistungspflicht der Krankenkasse ggf. gerichtlich durchsetzen $^{2}$. Der Sozialhilfeträger ist dann auch zur Zahlung höherer Kosten verpflichtet ${ }^{3}$.

Neben den Pflegeleistungen i.S. des SGB XI (insbesondere Grundpflegeleistungen i. S. des $\$ 14$ SGB XI) könnten

Rechtsanwalt Dr. iur. Kyrill Makoski,

Fachanwalt für Medizinrecht, Möller Partner,

Pfeifferstraße 6, 40625 Düsseldorf, Deutschland aber auch Leistungen der Behandlungspflege i.S. des $\$ 37$ Abs. 2 S. 1 SGB V fällig werden; in diesem Fall wäre dann die Krankenkasse leistungspflichtig ${ }^{4}$. Diese Leistungen sind i.d.R. nicht nach $\int 43$ a SGB XI von der Pflegeversicherung abgedeckt ${ }^{5}$ oder vom Heimbetreiber nach $\$ 75$ Abs. 5 SGB XII zu erbringen, da diese Vorschrift nur Leistungen nach SGB XI umfasst ${ }^{6}$.

Der Gesetzgeber hat 2008 durch eine Gesetzesänderung klargestellt, dass Leistungen der Behandlungspflege zu Lasten der Krankenkasse auch in betreuten Wohnformen und Werkstätten für Behinderte (d.h. Einrichtungen nach $₫ 43$ a SGB XI) erbracht werden können ${ }^{7}$. Zur Konkretisierung des Leistungsortes kann auf die Richtlinien des Gemeinsamen Bundesausschusses (GBA) über die Verordnung von häuslicher Krankenpflege in der vertragsärztlichen Versorgung nach $\$ 92$ Abs. 1 S. 2 Nr. 6 und Abs. 7 SGB V (HKP-RL) (zul. geänd. 21.10.2010, BAnz. 2011, S. 140) zurückgegriffen werden. Darin ist in $\$ 1$ Abs. 6 HKP-RL der Leistungsanspruch für die Dauer des Aufenthaltes in Krankenhäusern oder Heimen, in denen die Behandlungspflege durch den jeweiligen Träger gewährt wird, ausgeschlossen. $\$ 1$ Abs. 7 HKP-RL beinhaltet Ausnahmen für Pflegeleistungen in Werkstätten für behinderte Menschen oder Pflegeheimen. Ein zunächst in $\$ 1$ Abs. 6 HKP-RL vorgesehener Ausschluss für Einrichtungen der Eingliederungshilfe wurde auf Intervention des BMG entfernt, so dass jeweils eine Einzelfallentscheidung getroffen werden muss ${ }^{8}$. Durch die Änderung der HKP-RL sind die Bemühungen der Rechtsprechung, Werkstätten für Behinderte oder Wohnheime als ,,anderen geeigneten Ort“ zu definieren ${ }^{9}$, entbehrlich geworden. Die Frage eines „,eigenen Haushalts" ist nicht mehr entscheidend für die Kostentragung $^{10}$. Allerdings wurde die Einstandspflicht der Krankenkasse bei Heimen i.S. des HeimG verneint ${ }^{11}$. Teilweise wurde auf das Leistungsangebot des Heims abgestellt ${ }^{12}$; diese Auffassung ist auch vor dem Hintergrund von $\$ 1$ Abs. 6 HKP-RL zutreffend.

Das LSG hat in dieser Entscheidung die Verantwortung des Betreibers der Einrichtung betont. Wenn dieser in der Leistungsvereinbarung bestimmte Pflegetätigkeiten $\mathrm{zu}$ sichert, muss er organisatorisch sicherstellen, dass der gesamte Leistungsumfang bereit steht. Notfalls müssen der Dienstplan geändert oder zusätzliches Personal eingestellt werden. Wenn diese Maßnahmen aber nicht mehr ausreichen, muss der Betreiber darauf hinwirken, dass der Betroffene in eine geeignete Einrichtung verlegt wird.

1) Über $\$ 9$ Abs. 2 SGB XII hinaus, s. Wahrendorf, in: Grube/Wahrendorf (Hrsg.), SGB XII, 3. Aufl. 2010, \$55, Rdnr. 3.

2) Scheider, in: Schellhorn/Jirasek/Seipp (Hrsg.), SGB XII, 18. Aufl. 2010, $\$ 55$, Rdnr. 5.

3) BSG, Urt. v. 2.2.2010 - B 8 SO 20/08 R -.

4) LSG Hamburg, Beschl. v. 12.11.2009 - L 1 B 202/09 ER KR -

5) SG Stade, Urt. v. 30.3.2011 - S 19 SO 7/07 -, ZfSH/SGb 2011, 615

6) Flint, in: Grube/Wahrendorf (Hrsg.), SGB XII, 3. Aufl. 2010, $\$ 75$, Rdnr. 51; a. A. SG Stralsund, Urt. v. 10.8.2012 - S 3 KR 78/10 -, RdLH 2013, 10.

7) Rixen, in: Becker/Kingreen (Hrsg.), SGB V, 3. Aufl. 2012, \$37, Rdnr. 3; Ricken, in: Eichenhofer/Wenner (Hrsg.), SGB V, 2013, $\$ 37$, Rdnr. 4.

8) Weber, NZS 2011, 650, 652.

9) Vgl. LSG Sa.-Anh., Beschl. v. 26.8.2010 - L 8 SO 4/10 B ER -, PflR 2011, 93

10) SG Lüneburg, Urt. v. 6.12.2006 - S 22 SO 167/06 -, Sozialrecht aktuell 2007, 181.

11) LSG Nieders.-Bremen, Urt. v. 23.4.2009 - L 8 SO 1/07 -; a. A LSG Hamburg, Beschl. v. 12.11.2009 - L 1 B 202/09 ER KR 12) LSG Sa.-Anh., Beschl. v. 22.11.2011 - L 10 KR 32/11 B ER-. 